\section{P63 TREATMENT OF PELVIC INFLAMMATORY DISEASE} USING SHORT-COURSE AZITHROMYCIN

doi:10.1136/sextrans-2012-050601c.63

${ }^{1} \mathrm{~L}$ Cunningham, ${ }^{*}{ }^{1} \mathrm{~A}$ Murray, ${ }^{2} \mathrm{P} \mathrm{J}$ Horner. ${ }^{1}$ University Hospitals Bristol NHS Foundation Trust, Bristol, UK; ${ }^{2}$ University Hospitals Bristol NHS Foundation Trust/University of Bristol, Bristol, UK

Background In our centre, a novel 5-day regimen of azithromycin (1 g stat then $500 \mathrm{mg}$ daily for 4 days with or without metronidazole and/or ceftriaxone) is used and has been approved regionally. This is administered as an alternative regimen in selected cases of pelvic inflammatory disease (PID), usually those with milder symptoms and signs.

Aims and Objectives We assessed the use and efficacy of short course azithromycin in PID. We also assessed follow-up rates and adherence for all cases of PID.

Methods Retrospective case note review of 129 female patients coded as PID during the period 1 July to 31 December 2011. Data were collected and transferred to Excel for analysis.

Results 109/129 (84.5\%) of women were diagnosed with PID based on symptoms and signs as described in the BASHH PID guideline. Chlamydia was subsequently diagnosed in 10/108 (9.3\%) and gonorrhoea in 5/108 (4.6\%). Chlamydia antibody titres of $>1$ in 256 were found in $20 / 65$ (30.8\%) of patients. Taking into account missing data, 39/51 (76.5\%) patients completed the antibiotic course and 64/ $78(82 \%)$ reported full or partial resolution of symptoms. 14/85 $(16.5 \%)$ required further PID treatment, eight of whom had sexual intercourse with an untreated partner (see abstract P63 table 1).

Discussion In this study, 32/109 (29.4\%) women were treated for PID with azithromycin either in combination or alone. Overall, the majority of women completed treatment and reported a full or partial improvement in symptoms. Follow-up rates were good but documentation was inadequate. Comparisons between regimens are limited due to missing data. Further work will look at novel follow-up methods such as routine telephone contact as well as standardisation of follow-up documentation.

Abstract P63 Table 1 Antibiotic regime for PID treatment and response

\begin{tabular}{lllllll}
\hline $\begin{array}{l}\text { Principle } \\
\text { antimicrobial }\end{array}$ & $\begin{array}{l}\text { Treated } \\
(\%)\end{array}$ & $\begin{array}{l}\text { Follow-up } \\
\text { attended } \\
(\%)\end{array}$ & $\begin{array}{l}\text { Completed } \\
\text { course }\end{array}$ & & $\begin{array}{l}\text { Full/partial } \\
\text { clinical } \\
\text { response }\end{array}$ & \\
\hline & & & & $\begin{array}{l}\text { Missing } \\
\text { data }\end{array}$ & $\begin{array}{l}\text { Missing } \\
\text { data }\end{array}$ \\
Azithromycin & $32(29.4)$ & 21 & $5 / 8$ & 13 & $14 / 19$ & 2 \\
Ofloxacin & $71(65.1)$ & 59 & $32 / 40$ & 19 & $46 / 55$ & 4 \\
Doxycycline & $2(1.8)$ & 2 & $0 / 2$ & 1 & 2 & 0 \\
Other & $4(3.7)$ & 3 & 2 & 1 & 2 & 1 \\
Total & 109 & $85(78)$ & 39 & 34 & 64 & 7 \\
\hline
\end{tabular}

P64

HIGH RATES OF RECTAL CHLAMYDIA TRACHOMATIS IN WOMEN REPORTING ANAL SEX IN THE GUM CLINIC

doi:10.1136/sextrans-2012-050601c.64

S Simon, ${ }^{*}$ M Hassan-Ibrahim, S Soni. Brighton and Sussex University Hospitals NHS Trust, Brighton, UK

Background Nucleic acid amplification (NAAT) testing for Neisseria gonorrhoeae (GC) and Chlamydia trachomatis (CT) is now widely available but not licensed for use at non-genital sites. Despite this, high rates of pharyngeal and rectal GC and CT have been demonstrated in MSM. Currently there is little data on the prevalence of infection at these sites in women reporting oral and anal sex.

Aim To determine the prevalence of pharyngeal and rectal CT and GC in women attending GUM.
Methods Between 1 June and 31 October 2011 all women reporting oral and anal sex were offered pharyngeal and blind rectal swabs as well as cervical testing for CT and GC. Samples were analysed using the BD ProbeTecTM CT/GC Ox Amplified DNA assay in the BD ViperTM system. All women with positive GC NAAT results had swabs sent for GC culture and sensitivities.

Results Median age was 25 years (IOR 21-31) and most women were of white ethnicity. 2235 swabs were taken, 124 were excluded due to incomplete labelling. 2111 women had non-genital sampling: 165 rectal swabs only, 1652 pharyngeal swabs only, 147 both sites. In nine and five women the pharynx and rectum were the sole sites of CT infection respectively. In two women, the pharynx was the sole site of GC infection. All women were asymptomatic at throat and rectum (see abstract P64 table 1).

Discussion Rectal CT rates were high among women reporting anal sex. As the antibiotic treatment of cervical and rectal CT differs, this pool of infection could remain untreated. We recommend routinely asking about anal sex and testing where appropriate. Rates of pharyngeal CT were low but higher than GC overall. Although 11 women had pharyngeal CT/GC only, and would have been missed, the cost of detecting one of these infections was $£ 1727$ and this has precluded ongoing screening.

Abstract P64 Table 1 CT and GC prevalence at different sites

\begin{tabular}{lccc}
\hline & Pharynx & Rectum & \multicolumn{1}{l}{ Cervix } \\
\hline CT NAAT & $1.3 \%(23 / 1799)$ & $7.1 \%(22 / 312)$ & $6.1 \%(194 / 3073)$ \\
GC NAAT & $0.28 \%(5 / 1799)$ & $0.32 \%(1 / 312)$ & $0.49 \%(15 / 3073)$ \\
GC Culture & $0.17 \%(3 / 1799)$ & $0.32 \%(1 / 312)$ & $0.36 \%(11 / 3073)$ \\
\hline
\end{tabular}

\section{P65 SEROLOGICAL RESPONSE BETWEEN HIV-POSITIVE AND NEGATIVE COHORTS TREATED FOR EARLY SYPHILIS}

doi:10.1136/sextrans-2012-050601c.65

R Dhairyawan, ${ }^{*}$ A Almeida, M Gunathilake, B Goh. Barts and the London NHS Trust, London, UK

Introduction Successful treatment response for early syphilis involves resolution of signs and a fourfold decrease in RPR/VDRL or sero-reversion between 3 and 6 months. However, in the HIVpositive population the serological response can be unusual.

Aims To compare serological response after treatment of early syphilis between HIV-positive and negative cohorts.

Methods Retrospective cohort study of 100 HIV positive (HP) and 92 negative (HN) patients with early syphilis at an inner city GUM clinic from 2002 to 2011. Data were collected on demographics, stage of syphilis, treatment and serological response.

Results Both HP and HN cohorts were mainly MSM (72 HN, 96 $\mathrm{HP}$ ) and Caucasian (HN 76, HP 78). Median age 33 (range 20-63 years) in the HN and 37 (range 19-59 years) in the HP. Stage of syphilis: HN group, 22/92 primary, 26 secondary and 44 early latent (EL). HP group: 26/100 primary, 34 secondary and $39 \mathrm{EL}$. The $\mathrm{HN}$ group were treated with the standard regimen for their stage of disease. 49/100 of the HP group received treatment for neurosyphilis. 25/92 HN and 14/100 HP were lost to follow-up following treatment. Excluding these patients, by 6 months, 18/20 (90\%) of the HP group with primary syphilis sero-reverted or had a fourfold decrease in RPR/VDRL compared to $17 / 17$ (100\%) of the HN. By 6 months, 51/55 (93\%) of the HP group with secondary/EL syphilis had sero-reverted or had a fourfold decrease in RPR/VDRL compared to $41 / 45$ (91\%) of the HN. Median CD4 count in the HP group was 380 cells/ $\mu$ prior to treatment and 518 after treatment. There were 4 reinfections in the HN group and 10 in the HP.

Conclusions There were high rates of serological treatment success for early syphilis in both groups. However, the HP group were less 